\title{
Short-Term Oral Administration of Mesoporous Silica Nanoparticles Potentially Induced Colon Inflammation in Rats Through Alteration of Gut Microbiota
}

This article was published in the following Dove Press journal:

International Journal of Nanomedicine

Yue $\mathrm{Yu}^{\mathrm{l}, *}$

Zhou Wang'**

Ran Wang'

Jing Jin ${ }^{2}$

Yi Zhun Zhu (D) ${ }^{1,3}$

'State Key Laboratory of Quality Research in Chinese Medicine \& School of Pharmacy, Macau University of Science and Technology, Taipa, Macau SAR, People's Republic of China; ${ }^{2}$ Institute of Material Medica, Chinese Academy of Medical Sciences, Beijing, People's Republic of China; ${ }^{3}$ Shanghai Key Laboratory of Bioactive Small Molecules \& School of Pharmacy, Fudan University, Shanghai, People's Republic of China

*These authors contributed equally to this work

\begin{abstract}
Purpose: Mesoporous silica (MSNs) have attracted considerable attention for its application in the field of drug delivery and biomedicine due to its high surface area, large pore volume, and low toxicity. Recently, numerous studies revealed that gut microbiota is of critical relevance to host health. However, the toxicological studies of MSNs were mainly based on the degradation, biodistribution, and excretion in mammalian after oral administration for now. Here in this study, we explored the impacts of oral administration of three kinds of MSNs on gut microbiota in rats to assess its potential toxicity.
\end{abstract}

Methods: Forty rats were divided into four groups: control group; Mobil Composition of Matter No. 41 type mesoporous silica (MCM-41) group; Santa Barbara Amorphous-15 type mesoporous silica (SBA-15) group, and biodegradable dendritic center-radial mesoporous silica nanoparticle (DMSN) group. Fecal samples were collected 3 days and 7 days after the intake of MSNs and analyzed with high throughput sequencing. Gastric tissues in rats were obtained after dissection for the histological study.

Results: Three different MSNs (MCM-41, SBA-15, and DMSN) were successfully prepared in this study. The pore size of three MSNs was calculated similarly as $(3.54 \pm 0.15) \mathrm{nm},(3.48 \pm 0.21)$ $\mathrm{nm}$, and $(3.45 \pm 0.17) \mathrm{nm}$ according to the BET \& BJH model, respectively, while the particle size of MCM-41, SBA-15 and DMSN was around $209.2 \mathrm{~nm}, 1349.56 \mathrm{~nm}$, and $244.4 \mathrm{~nm}$, respectively. In the gene analysis of $16 \mathrm{~S}$ rRNA, no significant changes in the diversity and richness were found between groups, while Verrucomicrobia decreased and Candidatus Saccharibacteria increased in MCM-41 treated groups. Meanwhile, no inflammatory and erosion symptoms were observed in the morphological analysis of the colons, except the MCM-41 treated group.

Conclusion: Three different MSNs, MCM-41, SBA-15, and DMSN were successfully prepared, and this study firstly suggested the impact of MSNs on the gut microbiota, and further revealing the potential pro-inflammatory effects of oral administration of MCM-41 was possibly through the changing of gut microbiota.

Keywords: gut microbiota, mesoporous silica, MCM-41, SBA-15, dendritic mesoporous silica nanoparticle

\section{Introduction}

With the increasing development of nanotechnology, nanoparticles have been widely used to conduct more specific and efficient treatments towards complex diseases. ${ }^{1}$ On the one hand, nanoparticles used as drug carrier could deliver a drug
Correspondence: Yi Zhun Zhu

Macau University of Science and

Technology, Block E, Avenida Wai Long,

Republic of China

Tel + 8685388972880

Fax +868532882 3575

Email yzzhu@must.edu.mo
International Journal of Nanomedicine 2021:16 881-893

881

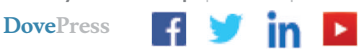

http://doi.org/10.2147/un.S295575 
in a sustained and controlled manner, on the other hand, it could also improve the bioavailability in vivo, the stability of the cargo, and the convenience for administration, overcoming some of the typical limitations of systemic treatments.

Among all the widely used nano-vehicles, mesoporous silica (MSNs) has been proven to be effective drug carriers for oral delivery, especially for water-insoluble drugs, inducing significantly enhanced oral bioavailability. With the high surface area and large pore volume of MSNs, it would be able to encapsulate various therapeutic agents with high loading capacity. Also, it would be easy for the modification of the functional group on MSNs' surface to achieve the long period release of therapeutic agents. ${ }^{2,3}$ For example, Shah's group ${ }^{4}$ fabricated a Velpatasvir (VLP) loaded MSNs with good biocompatibility, and this MSNs based drug delivery system showed prolonged VLP release both in vitro and in vivo. Meantime, a study by Yang et $\mathrm{al}^{5}$ revealed a preparation of Breviscapine (BRE) loaded MSNs, which showed higher dissolution rate and higher oral absorption than BRE powder. In a work by An's group, ${ }^{6}$ a core-shell type MSNs was developed for fluorescent imaging, stimuli-responsive drug release, magnetic separation, antibody targeting, and chemophotothermal therapeutics was also reported.

Given the growing use of MSNs in the drug delivery system, ${ }^{7}$ the safety and toxicity of the chronic exposure to MSNs in mammalian should be a major concern for preclinical evaluation. It should be noted that the surface area, surface charge, particle size, porous structure, and prepare method all matters when evaluating the toxicological properties of MSNs. ${ }^{8}$ In the study by Fu et $\mathrm{al}^{9}$ mice were single administrated with MSNs through different exposure routes (intravenous injection, hypodermic injection, intramuscular injection and oral administration). On day 7, it was found that through pathological examinations, MSNs possessed good tissue biocompatibility after oral and intravenous injection. $\mathrm{Li}$ et $\mathrm{al}^{10}$ performed a study of the toxicity after oral administration of MSNs with different aspect ratios $(1,1.75$, and 5$)$, while results revealed that higher aspect ratio showed lower biotoxicity in vivo.

The mammalian gastrointestinal tract is the main site for commensal bacteria. There are thousands of microorganisms inside mammalian body, which maintaining mammalian gastrointestinal homeostasis, maybe even play important role in the mammalian health. Some studies have been conducted on the influence of nanoparticles made on gut microbiota, mainly on food additives. For example, Chen et $\mathrm{al}^{11}$ reported that chronic co-exposure to titanium dioxide nanoparticles and bisphenol A could promote the development of pathogenic Lawsonia but depress the normal metabolic activity of Hyphomicrobium. Wilding et $\mathrm{al}^{12}$ found that with repeated ingested (28 days) of $\mathrm{Ag}$ nanoparticles of varied size and coating, it did not cause any alterations in the structure or diversity of the mice gut microbiome, though the Ag nanoparticles having antimicrobial properties in vitro.

The purpose of this study was to evaluate the gut microbial community profiles in a rat model following the administration of three kinds of MSNs, which were Mobil Composition of Matter No. 41 type mesoporous silica (MCM-41), Santa Barbara Amorphous-15 type mesoporous silica (SBA-15), and biodegradable dendritic center-radial mesoporous silica nanoparticle (DMSN).

\section{Materials and Methods Chemical Materials}

Cetyltrimethylammonium bromide (CTAB), cetyltrimethylammonium chloride (CTAC), PEO-PPO-PEO (P123), triethanolamine (TEA) and decahydronaphthalene (DHA) were purchased from Sigma-Aldrich (St. Louis, USA). Tetraethyl orthosilicate (TEOS) was provided by Aladdin Industrial Corporation (Shanghai, China); Sodium hydroxide, hydrochloric acid, acetonitrile, methanol, and anhydrous ethanol were purchased from Fuchen Chemicals Reagent Factory (Tianjin, China). The 1\% (w/v) agarose gels, TBE buffer (Tris-boric acid-EDTA), and ethidium bromide (EB) were obtained from MultiSciences (Hangzhou, China).

\section{Synthesis and Characterization of Nanoparticles}

The MCM-41 was synthesized via modified templating method, ${ }^{13}$ briefly, the mixture of $\mathrm{CTAB}, \mathrm{NaOH}$ and distilled water was mixed together and heated at $80{ }^{\circ} \mathrm{C}$ for 30 min. Afterwards, TEOS was added dropwise. The reaction was maintained at $80{ }^{\circ} \mathrm{C}$ for $2 \mathrm{~h}$. The resultant suspension was then centrifuged at $4000 \mathrm{rpm}$ for $15 \mathrm{~min}$. Washed the precipitation with distilled water, then dried at $45^{\circ} \mathrm{C}$ overnight. Finally, to further purify the particles, they were calcined at $550{ }^{\circ} \mathrm{C}$ for $5 \mathrm{~h}$.

The SBA-15 was synthesized via the method of Zhao et al. ${ }^{14}$ Typically, P123 was firstly dissolved in the mixture of deionized water and $\mathrm{HCl}$, with further the addition of TEOS and stirred at $40{ }^{\circ} \mathrm{C}$ for $24 \mathrm{~h}$. Finally, the product was crystallized at $120{ }^{\circ} \mathrm{C}$ for another 24 
h. The sample was then washed and calcined at $550^{\circ} \mathrm{C}$ for $5 \mathrm{~h}$.

The DMSN was synthesized via novel biphase stratification approach with little modification. ${ }^{15}$ Briefly, CTAC solution and TEA were added to deionized water with stirring at $60{ }^{\circ} \mathrm{C}$ for $1 \mathrm{~h}$. TEOS-DHA solution was then dropped and the reaction was kept magnetic stirring for another $12 \mathrm{~h}$. The mixture was then centrifugated, the precipitation was washed and calcinated at $550{ }^{\circ} \mathrm{C}$ for $5 \mathrm{~h}$.

The particle size of the nanoparticles was measured by Malvern Zetasizer Nano Series (Malvern, USA). The SEM (Nova Nano SEM, Philips, Netherlands) and TEM (JEM2100F High Resolution JEOL, Japan). The nitrogen adsorption-desorption was performed using a Micromeritics Tristar 3000 pore analyzer (Micromeritics, USA) to determine the pore size, pore volume, and specific surface area. The surface area was determined using the Brunauere Emmere Teller (BET) model, and the pore size distributions was calculated from the adsorption branch of the isotherm using the Barrette Joyner Halenda (BJH) model.

\section{Animals Experiment Design}

The Animal Care and Use Committee of Municipal Affairs Bureau of Macau approved all studies described herein (approval number AL010/DICV/SIS/2018), and experiment was conducted under the guidance of NIH Guide for the Care and Use of Laboratory Animals (8th edition). Neonatal Sprague-Dawley (SD) rats were purchased from the University of Hong Kong.

Forty male Sprague Dawley rats were housed in a 12 $\mathrm{h}$ light-dark cycle facility, and all the rats had free access to food and water. Rats were randomly divided into 4 groups, which were control, MCM-41 treated group, SBA-15 treated group and DMSN treated group, respectively. Each group containing of 10 healthy 8 -weeks-old rats weighting $200 \pm 20 \mathrm{~g}$, housed in 2 different cages (5 rats in one cage). All the rats were daily intragastrically with $50 \mathrm{mg} \cdot \mathrm{kg}^{-1} \mathrm{MSNs}$ for 7 days, dosage was consistent with reference published. ${ }^{10}$ The $\mathrm{CO}_{2}$-based euthanasia of rats was conducted under the American Veterinary Medical Association guidance.

\section{Pro-Inflammation Effects Examination}

Body weights of rats were recorded every day, and at the 7th day, rats were sacrificed, the colon samples were collected and thoroughly washed with PBS buffer to remove the residual intestinal content, and fixed in $10 \%$ phosphatebuffered formalin acetate, then embedded in paraffin for histological observation. The pathological change of rats after ingestion of MSNs was examined by H\&E staining.

Blood was collected on the same day from all groups, and serum samples were obtained by centrifuging and stored at $-40{ }^{\circ} \mathrm{C}$ until use. Serum TNF- $\alpha$ and IL- $1 \beta$ levels were measured using ELISA kits according to the manufacturer's instructions.

\section{DNA Extraction}

Total community genomic DNA extraction was performed using an E.Z.N.A. Soil DNA Kit (Omega, USA), following the manufacturer's instructions. The concentration of the DNA was measured using a Qubit 2.0 (Life, USA) to ensure that adequate amounts of high-quality genomic DNA had been extracted.

\section{I6S rRNA Gene Amplification by PCR}

Our target was the V3-V4 hypervariable region of the bacterial 16S rRNA gene. PCR was started immediately after the DNA was extracted. The 16S rRNAV3-V4 amplicon was amplified using KAPA HiFi Hot Start Ready Mix (2×) (TaKaRa Bio Inc., Japan). Two universals bacterial 16S rRNA gene amplicon PCR primers (PAGE purified) were used: the amplicon PCR forward primer (CCTACGGGNGGCWGCAG) and amplicon PCR reverse primer (GACTACHVGGGTATCTAATCC). The reaction was set up as follows: $2 \mu \mathrm{L}$ of microbial DNA (10 ng/ $\mu \mathrm{L}) ; 1 \mu \mathrm{L}$ of amplicon PCR forward primer $(10 \mu \mathrm{M}) ; 1 \mu \mathrm{L}$ of amplicon PCR reverse primer $(10 \mu \mathrm{M}) ; 15 \mu \mathrm{L}$ of $2 \times$ KAPA HiFi Hot Start Ready Mix. The plate was sealed, and PCR performed in a thermal instrument (Applied Biosystems 9700, USA) using the following program: 1 cycle of denaturing at 95 ${ }^{\circ} \mathrm{C}$ for $3 \mathrm{~min}$, which including first 5 cycles of denaturing at 95 ${ }^{\circ} \mathrm{C}$ for $30 \mathrm{~s}$, annealing at $45^{\circ} \mathrm{C}$ for $30 \mathrm{~s}$, elongation at $72{ }^{\circ} \mathrm{C}$ for $30 \mathrm{~s}$, then 20 cycles of denaturing at $95^{\circ} \mathrm{C}$ for $30 \mathrm{~s}$, annealing at $55^{\circ} \mathrm{C}$ for $30 \mathrm{~s}$, elongation at $72^{\circ} \mathrm{C}$ for $30 \mathrm{~s}$ and a final extension at $72{ }^{\circ} \mathrm{C}$ for $5 \mathrm{~min}$. The PCR products were checked using electrophoresis in $1 \%(\mathrm{w} / \mathrm{v})$ agarose gels in TBE buffer stained with EB and visualized under UV light.

\section{I6S Gene Library Construction, Quantification, and Sequencing}

AMPure XP beads were used to purify the free primers and primer dimer species in the amplicon product. Samples were delivered to Sangon BioTech (China) for library construction using universal Illumina adaptor and index. Before sequencing, the DNA concentration of each PCR product was determined using a Qubit ${ }^{\circledR} 2.0$ Green 
double-stranded DNA assay and it was quality controlled using an Agilent 2100bioanalyzer (Agilent, USA). Depending on coverage needs, all libraries can be pooled for one run. The amplicons from each reaction mixture were pooled in equimolar ratios based on their concentration. Sequencing was performed using the Illumina MiSeq system (Illumina, USA), according to the manufacturer's instructions.

\section{Sequence Processing}

After sequencing, data were collected as follows: (1) The two short Illumina readings were assembled by PEAR (v0.9.6) software according to the overlap and fastq files were processed to generate individual fasta and qual files, which then be analyzed by standard methods. (2) Sequences containing ambiguous bases and any longer than 480 base pairs (bp) were dislodged and those with a maximum homopolymer length of $6 \mathrm{bp}$ were allowed, ${ }^{16}$ while those with sequence short than 200bp were removed. (3) All identical sequences were merged into one. (4) Sequences were aligned according to a customized reference database. (5) The completeness of the index and the adaptor was checked and removed all the index and the adaptor sequence. (6) Noise was removed using the Pre.cluster tool. Chimeras were detected by using Chimera UCHIME. All the software was in the mothur package. The effective sequences of each sample were submitted to the RDP Classifier again to identify archaeal and bacterial sequences. The modified pipeline was described on the mothur website. Finally, all effective bacterial sequences without primers were submitted for downstream analysis. $^{17}$

\section{Bacterial Diversity and Taxonomic Analysis}

Bacterial diversity and richness were determined by sampling-based OTUs analysis and presented by the Chaol index, ACE index, Shannon index, and Simpson index, which were calculated using R program package "vegan". Bacterial taxonomic analyses and comparison at bacterial phylum level were conducted between groups using Wilcoxon rank sum test. Stool microbial characterization was analyzed by linear discriminant analysis (LDA) effect size (LEfSe).

\section{Statistical Analysis}

Statistical analyses of gut microbiome samples were performed using SPSS and GraphPad Prism (version 6.0) software packages. Differences in the pro-inflammatory cytokines among groups were determined using Student's $t$-test. Unpaired Mann-Whitney rank sum test (two-tailed) was used for comparisons of continuous variables between two groups. Violin plots were used to represent the data's mean at the center values, with error bars to indicate SD values. Spearman's rank correlation tests (two-tailed) were used to find significant correlations between two continuous variables. LEfSe was used to identify differentially abundant features between classes of samples. Unadjusted P-values 0.05 were considered significant for the Mann-Whitney rank sum test and Spearman's rank correlation tests. Statistical analyses were performed using SPSS V.20.0 for Windows.

\section{Results}

\section{Characterization of MSNs}

As shown in Figure 1, the bulk-shaped MCM-41 (Figure 1A), the rod-like shapes SBA-15 (Figure 1C) and the spherical morphology of DMSN (Figure 1E) could be observed under SEM, and the TEM further confirmed the pore channels in MCM-41, SBA-15 and DMSN (Figure 1B, D and F). Especially in the DMSN group, the central radially pore channel could be observed (Figure 1F). According to the $\mathrm{N}_{2}$ adsorption-desorption isotherms, the prepared MCM-41 (Figure 1G, red line) and SBA-15 (Figure 1G, blue line) exhibited a typical type-IV isotherm containing H1-type hysteresis, suggesting the mesoporous structure. Meanwhile, a capillary condensation step around $0.2<\mathrm{P} \cdot \mathrm{P}_{0}^{-1}<0.4$ could be observed, which indicated a mesopore size distribution (Figure 1G, green line). The pore size of MCM-41, SBA15 and DMSN was calculated as $(3.54 \pm 0.15) \mathrm{nm},(3.48 \pm$ $0.21) \mathrm{nm}$, and $(3.45 \pm 0.17) \mathrm{nm}$, while surface area was calculated as $417.85 \mathrm{~m}^{2} \cdot \mathrm{g}^{-1}, \quad 477.31 \mathrm{~m}^{2} \cdot \mathrm{g}^{-1}$, and $565.31 \mathrm{~m}^{2} \cdot \mathrm{g}^{-1}$ according to the BET \& BJH model, respectively (Figure 1H). The particle size of MCM-41, SBA-15 and DMSN was around $209.2 \mathrm{~nm}, 1349.56 \mathrm{~nm}$, and $244.4 \mathrm{~nm}$, respectively (Figure 1I). The zeta potential of the prepared MSNs (Figure 1J) indicated MCM-41, SBA-15, and DMSN with a negatively charged surface, and their absolute values were all around 25, which indicated a good dispersity of the prepared MSNs. 


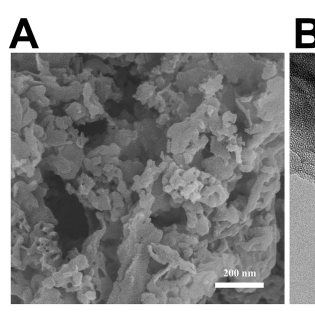

G

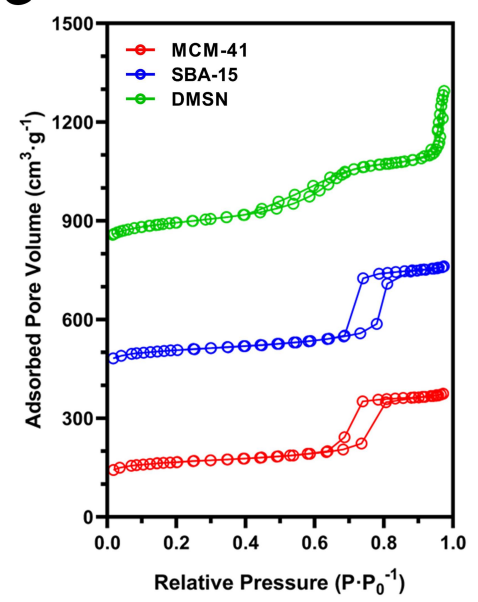

C

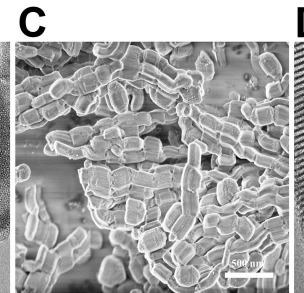

H

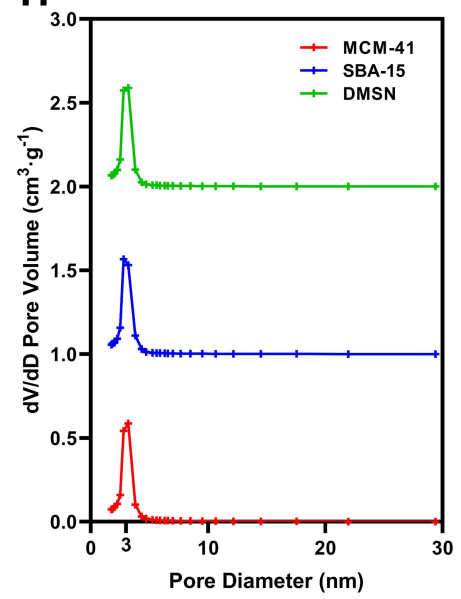

E

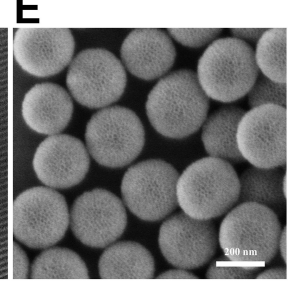

$\mathbf{F}$

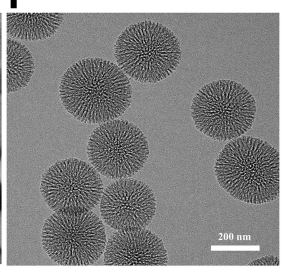

I

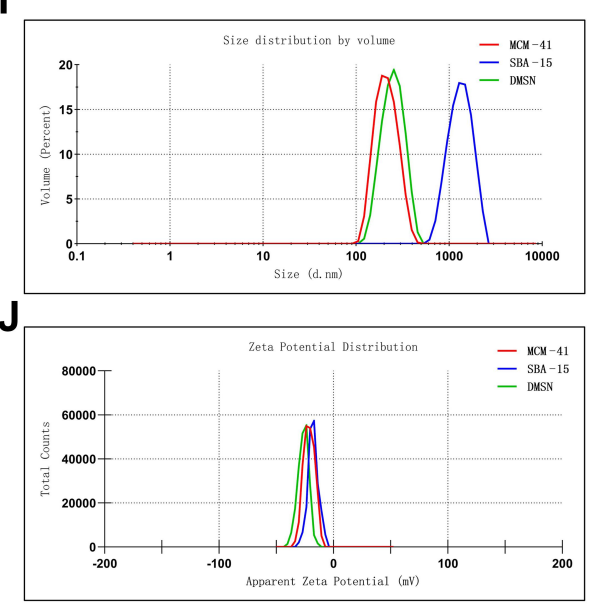

Figure I The characterization of MCM-4I, SBA-I5, and DMSN. (A, C and E) The representative SEM images and (B, D and F) TEM images of MCM-4I, SBA-I5, and DMSN. (G) The nitrogen adsorption-desorption isotherms and $(\mathbf{H})$ the pore size distribution were measured according to the BET \& BJH model. (I) The particle size and (J) zeta potential were measured.

\section{Potential Pro-Inflammation Effects After Oral Administration of MSNs}

The body weight, pro-inflammatory cytokines, and H\&E staining examination were used to evaluate the side effects of a 7-day oral administration of MSNs in rats. The body weight of rats in 4 different groups showed a similar increase tendency indicated a low toxicity effect
(Figure 2A). In the study of cytokines levels in serum, no significant difference was found among groups ( $p>0.05$ ), which revealed possibly a low proinflammatory effects of a 7-day oral administration of these three different MSNs (Figure 2B and C). To further investigate the potential pro-inflammatory effects after oral administration, the H\&E examination of rats' colons was
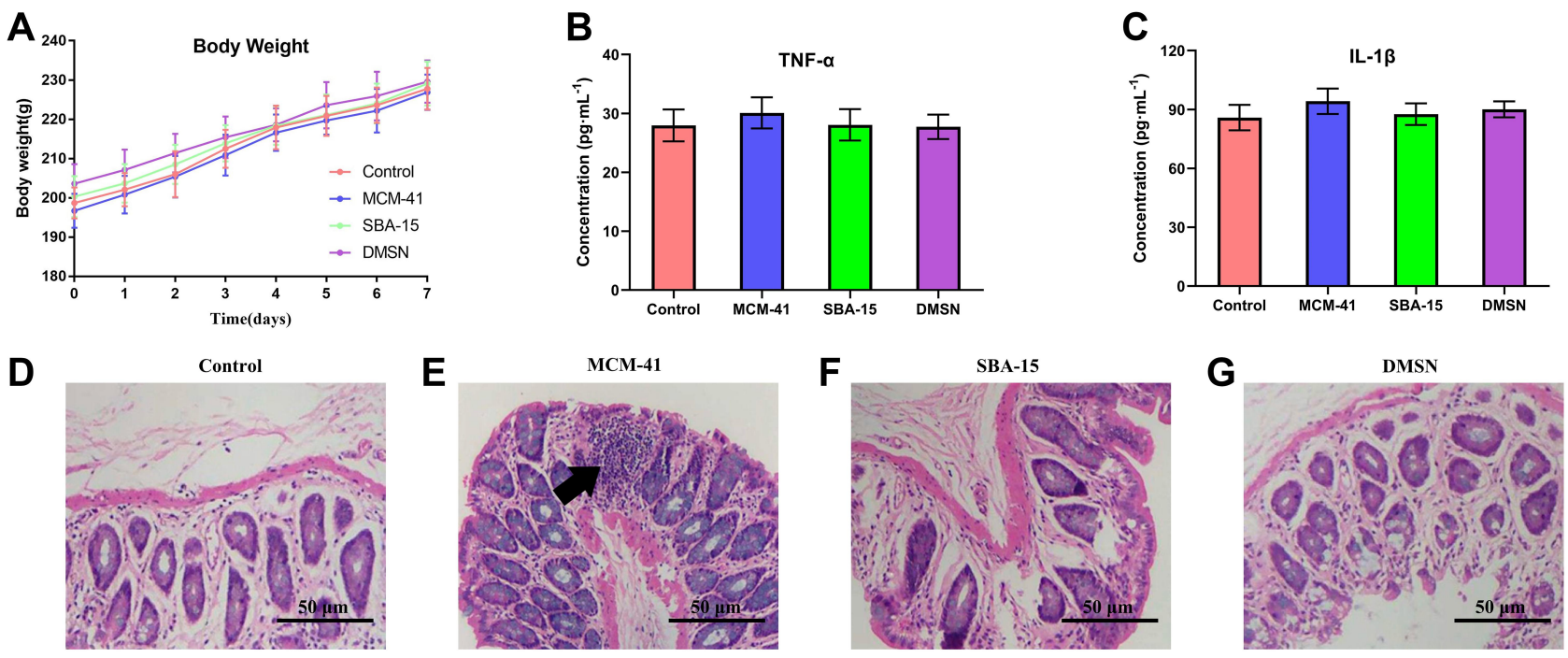

Figure 2 The potential pro-inflammation effects after various treatments within 7 days. (A) The body weight changes and the serum level of (B) TNF- $\alpha$ and (C) IL-I $\beta$ were measured. $(n=10$, mean $\pm S D)$. (D-G) The histology examination of colon via H\&E staining. Black arrow indicated the potential site of inflammation. 
conducted (Figure 2D-G). As shown, no severe inflammatory symptom observed in control, SBA-15 treated and DMSN treated groups, while interestingly, a slight inflammation could be observed in the MCM-41 treated group. To further reveal the potential pro-inflammatory effects of MCM-41 after oral administration, the gut microbiota of different treated group was then investigated.

\section{Microbiota Diversity}

A database consisting of $6,378,091$ total sequences were generated with a mean of $79,726 \pm 3678$ reads per sample. The range of the number of the reads for the samples was from 47,827 to 127,235 . There were up to 87,969 OTUs among all subjects. As shown in Figure 3, Chaol index and Abundance-based Coverage (ACE) index were measured for microbial richness assessment, and no significant difference was found between groups during the 7-day experimental period. Meanwhile, the Shannon and Simpson diversity indices were measured to compare the diversity of the gut microbiome between groups. Similarly, no significant difference was observed. Accordingly, no significant difference of the indices was observed at different time points (day 3 vs day 7) in each group. These results suggested that daily oral administration of neither MCM-41, SBA-15, nor DMSN showed no significant alteration of the richness and diversity of rats' gut microbiota within the 7-day experimental period.

\section{Impact of MSNs on Rat Gut Microbiota}

A database consisting of 6,378,091 total sequences were generated with a mean of $79,726 \pm 3678$ reads per sample. The range of the number of the reads for the samples was from 47,827 to 127,235 . There were up to 87,969 OTUs among all subjects. As shown in Figure 3, Chao1 index and Abundance-based Coverage (ACE) index were measured for microbial richness assessment, and no significant difference was found between groups during the 7-day experimental period. Meanwhile, the Shannon and Simpson diversity indices were measured to compare the diversity of the gut microbiome between groups. Similarly, no significant difference was observed. Accordingly, no significant difference of the indices was observed at different time points (day 3 vs day 7) in each group. These results suggested that daily oral administration of MCM41, SBA-15 and DMSN showed no significantly alteration of the biodiversity in rats' gut microbiota within the 7-day experimental period.
In taxonomy study, a total of 22 phyla were found, while two of the phyla, Bacteroidetes and Firmicutes, were predominant in the gut microbiota of all subjects. Meantime, the smaller populations included Verrucomicrobia, Cyanobacteria, Proteobacteria, etc., were also detected. Circos graph, as shown in Figure 4, is effective in visualizing differences of microbiota between groups, not only reflecting the proportion of the dominant phyla in each group, but also reflecting the distribution of each dominant phylum in the different groups with different width of each side of the connecting bands between the sample groups and microbiota compositions. Herein, the Circos graph showed the alteration of the gut microbiota in each group was not obviously noticeable. Interestingly, weighted UniFrac principal coordinate analysis (PCoA), a $\beta$-diversity measure using phylogenetic information to compare environmental samples, showed the control group clustered away from other groups at day 7 whereas no dramatic separation between control and other groups was observed at day 3 (Figure 5).

To further investigate whether there is an alteration in some specific phyla at day 7 , based on the taxonomy data, distinguished taxa between groups were identified by performing Linear discriminant analysis Effect Size (LEfSe). At phylum level, as shown in LEfSe circular cladogram (Figure 6A) and LDA score (Figure 6B), Verrucomicrobia, Candidatus Saccharibacteria were shown to be predominant in control and MCM-41 groups, respectively.

As shown in Figure 7, one-way ANOVA bar plot was made to demonstrate the alteration of the predominant phyla between groups. Significantly higher Verrucomicrobia was detected in control group, and significantly higher Candidatus Saccharibacteria was detected in MCM-41 group.

\section{Discussion}

MSNs have been demonstrated to be an excellent drug delivery system due to their adjustable pore size, various particle size and large surface area, and they have also been reported to be a potential candidate drug carrier in the treatment of bacterial infection. ${ }^{18-20}$ Although MSNs have been proved to be biocompatible and biodegradable, owing to MSNs' various characteristics, the conclusions of their systematic toxicity in previous studies were found varied. Hence, it is still controversy whether the use of MSNs as drug carrier is safe. Most of the reports showed that the MSNs preferentially accumulated in the liver and 

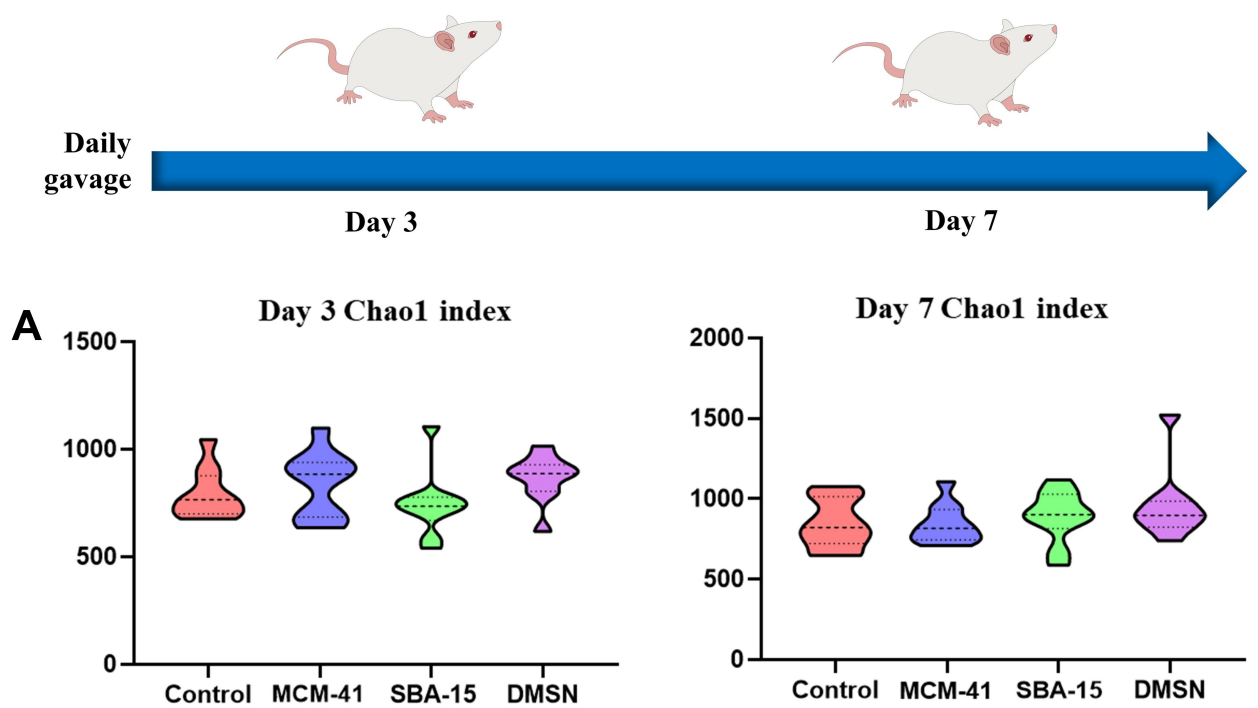

B
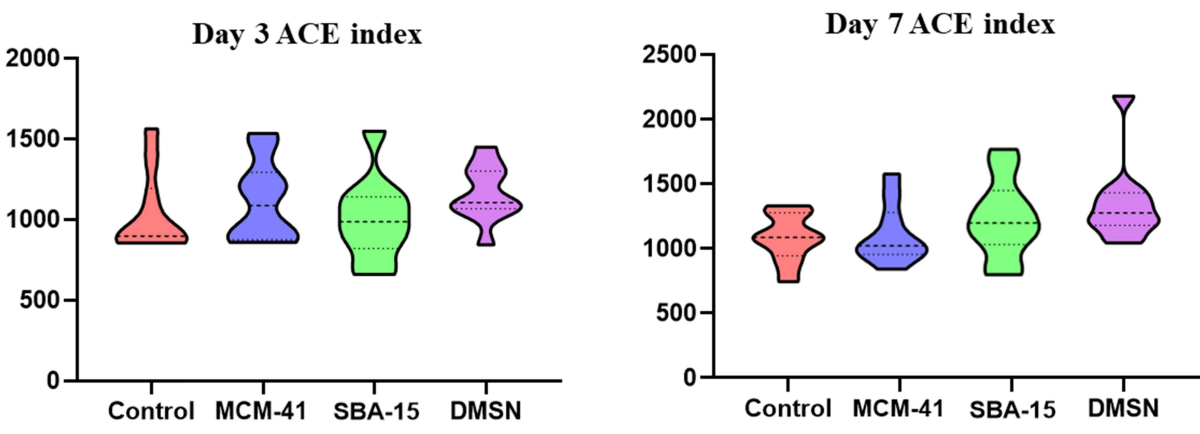

C

Day 3 Shannon index

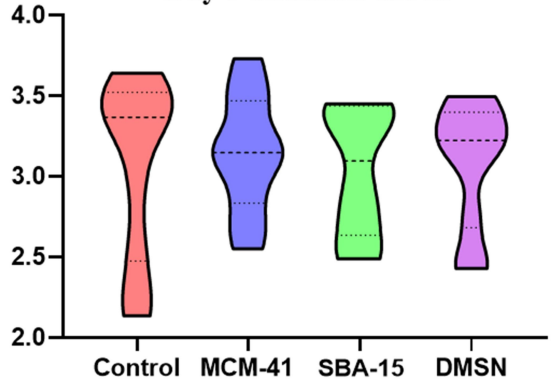

Day 7 Shannon index

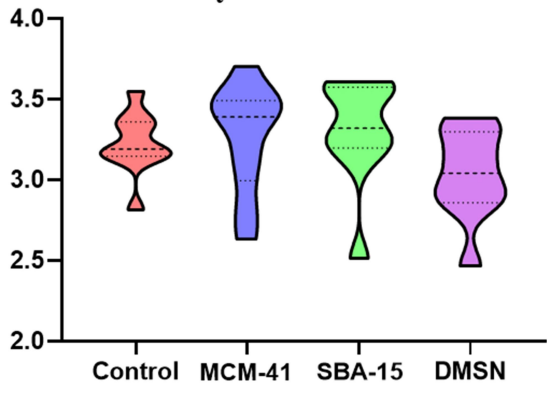

D
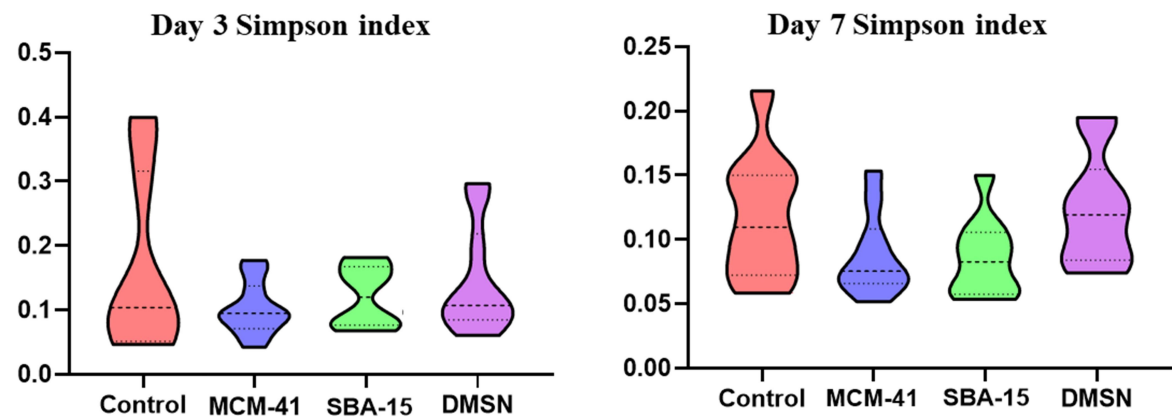

Figure 3 The diversity of gut microbiota. Results were shown as violin plot, the width of the shaded area represents the proportion of the data. (A) The Chaol index; (B) ACE index; (C) The Shannon index; (D) Simpson index at day 3 and day 7. $(n=10$, mean \pm SD). 

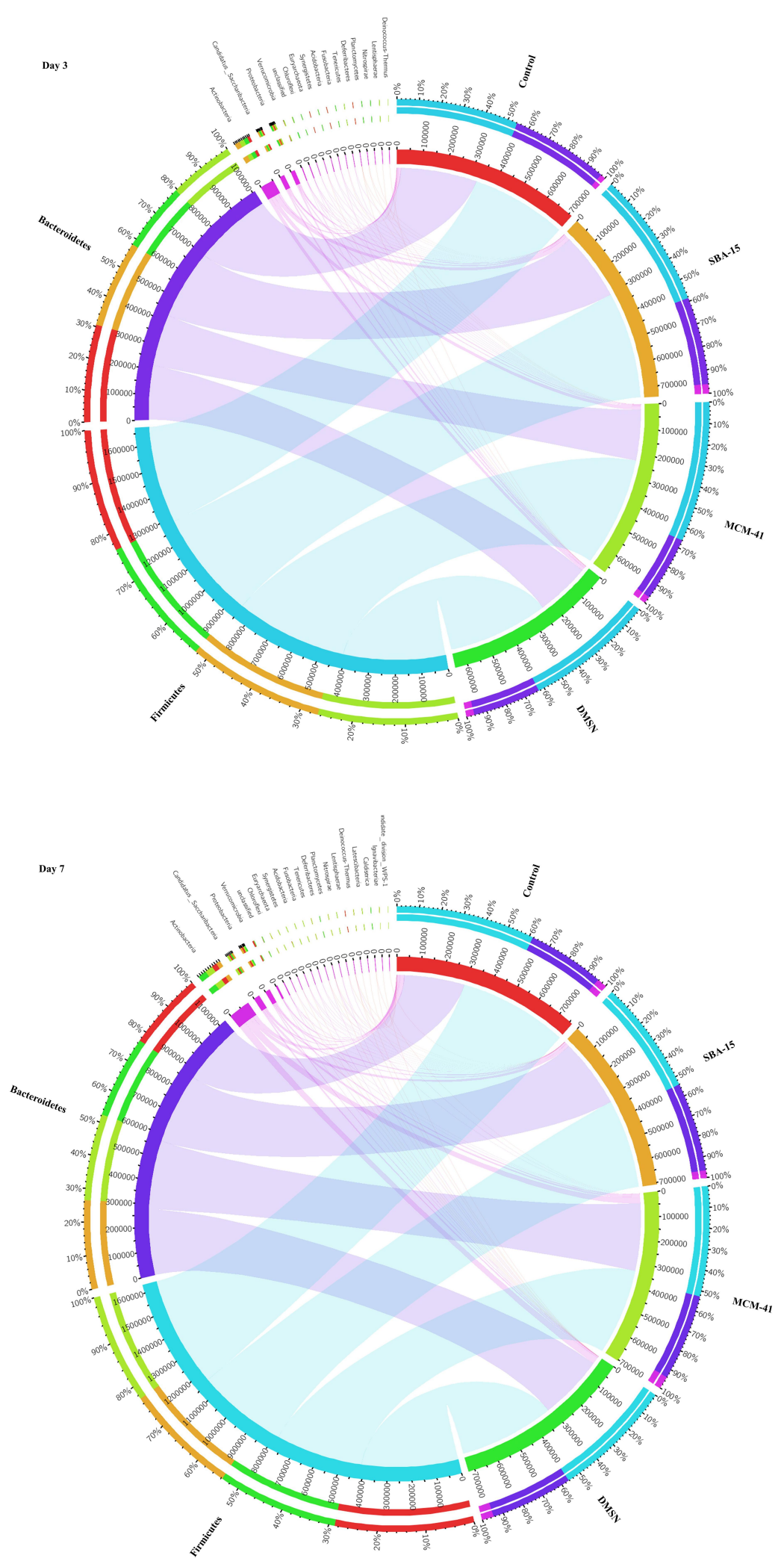

Figure 4 The differences of microbiota between groups. The Circos graphs at day 3 and day7, while the left semicircle represents the phyla composition of each group, and the right semicircle indicates the distribution of each phylum in the different groups. $(n=10)$. 

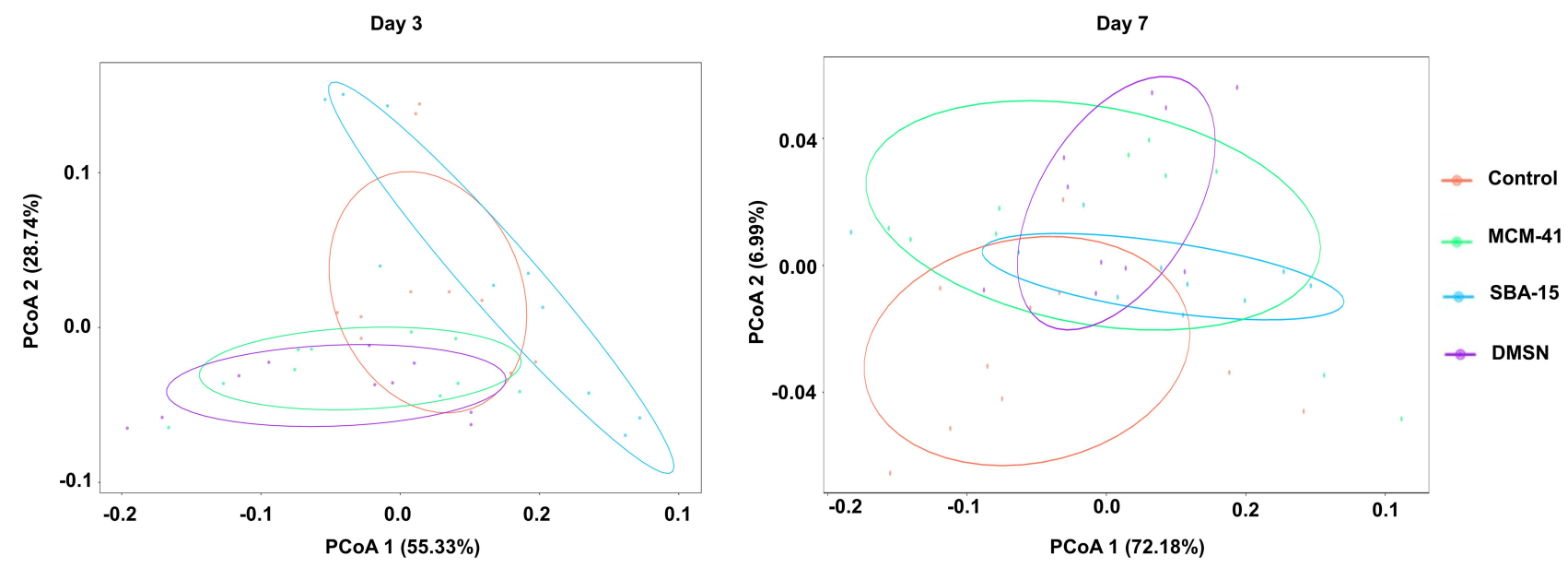

Figure 5 PCoA of the different treated groups at the OTU level at day 3 and day $7(n=10)$.

spleen after oral administration, ${ }^{21,22}$ but its influence on gut microbiota after oral administration remains unclear.

In this study, we successfully prepared three different MSNs, which were MCM-41, SBA-15, and DMSN, and further demonstrated for the first time that the impact of orally administration of MSNs with various size and shapes on the gut microbiota. A total of 22 phyla, 42 classes, 69 orders, 139 families and 265 genera were identified in all fecal samples. Meanwhile, we found that the diversity and richness of the rats' gut microbiota did not differ greatly at different time point (day 3 and day 7) in each group. Moreover, no significant changes in the diversity and richness were found between groups, while Verrucomicrobia and Candidatus Saccharibacteria (formerly known as candidate phylum TM7) varied. To the best of our knowledge, this was the first study to investigate the influence of MSNs with various size and shape on gut microbiota of rats.

The parameters like surface charge, ${ }^{23}$ pore size, ${ }^{23,24}$ pore channel structure, ${ }^{15}$ particle shape, ${ }^{10,25}$ particle size, ${ }^{26}$ and even exposure routes ${ }^{9}$ all matters when investigating the toxicity of MSNs. Herein, we prepared three MSNs with same surface charge (negatively charged) and similar pore size $(\sim 3 \mathrm{~nm})$, with further administrated with the same exposure route (i.g.). Lower aspect ratio, ${ }^{10,25}$ irregular pore channel structure, ${ }^{15}$ and smaller particle size ${ }^{26}$ might have higher toxicity and induce inflammation as reported. Wherein, MCM-41 has the lowest aspect ratio, smallest particle size, and the most irregular pore channel structure among these three MSNs, that was the potential reason why the MCM-41 could induce inflammation in colon.

As a large proportion of the mammalian intestinal microbes cannot be cultured using standard microbiological techniques, the $16 \mathrm{~S}$ rRNA gene sequencing has been employed in our study for the V3-V4 regions of 16S rRNA gene could provide abundant available information for the taxonomic classification of microbial communities from mammalian intestinal flora samples, and were well applied in many previous studies. ${ }^{27-29}$

In previous studies, the toxicity of silica-based nanomaterials has been well studied. ${ }^{23,30-32}$ Many researchers demonstrated that nanoparticles with smaller size might increase the toxicity or change the mechanism by which they induce toxicity. Studies of the toxicity of MSNs are complex due to the size- and shape-dependent properties of MSNs which might show different levels of toxicity both in vitro and in vivo. Considering the MCM-41 and SBA-15 have been well studied as drug carrier, and their toxicity have been fully studied except for the influence on gut microbiota, we prepared MCM-41 and SBA-15 with similar pore size (around $3.5 \mathrm{~nm}$ ), and at meantime, a DMSN with pore size around $3.5 \mathrm{~nm}$ was also prepared, the DMSN was chosen for its biodegradability as reported. $^{15}$

Although the diversity and richness of different groups did not change significantly during the 7 days, the abundance of less abundant Verrucomicrobia was found significantly decreased and Candidatus Saccharibacteria was found significantly increased in MCM-41 treated group at day 7. For Verrucomicrobia, as reported, has been proposed to be a potential biomarker in many diseases and has a close correlation with mammalian metabolism. For example, a decreased abundance of Verrucomicrobia could be observed in accelerated aging rats and in obese type 2 diabetes patients. Additionally, a bloom of Verrucomicrobia was observed in mice treated with 
A

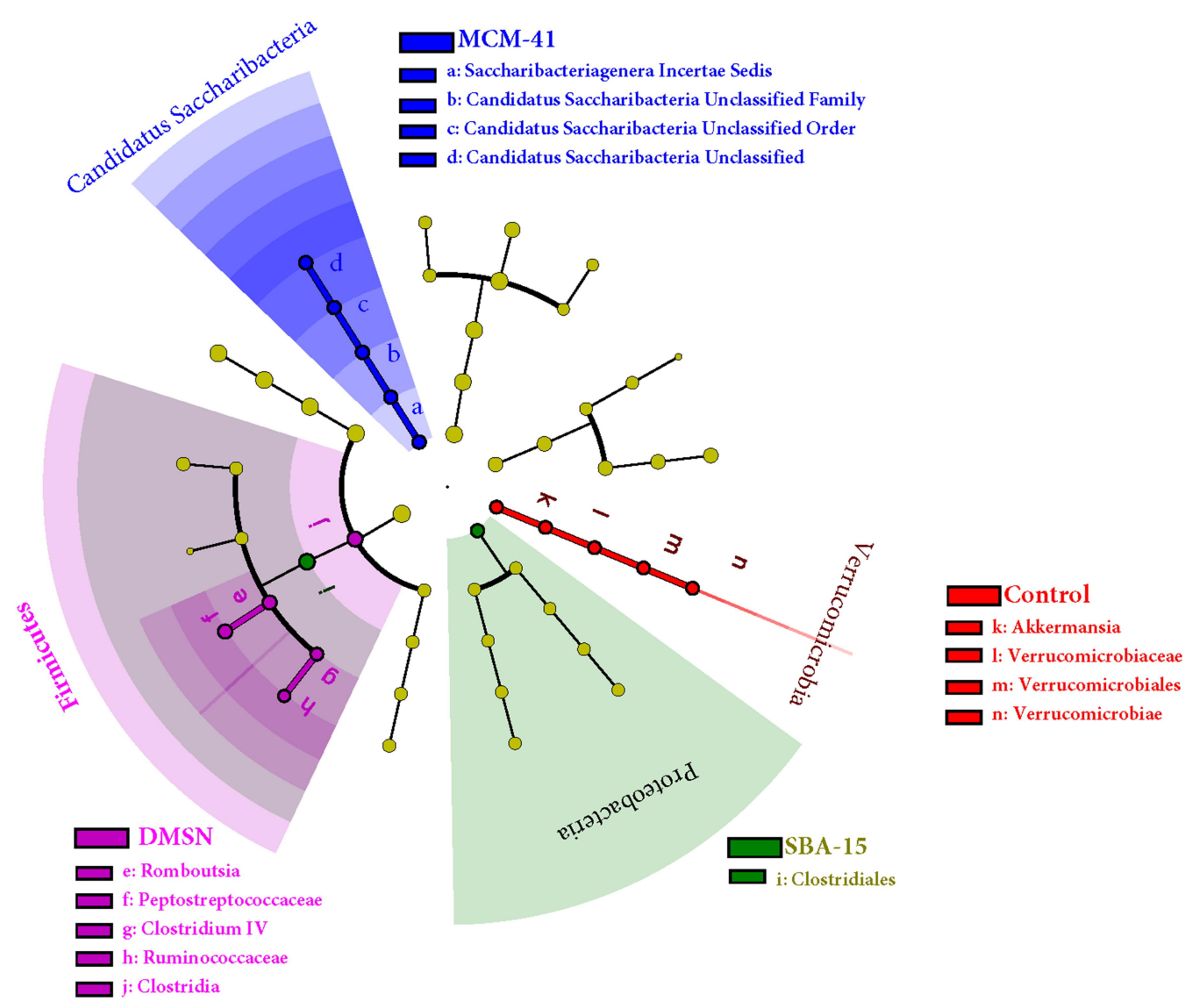

B

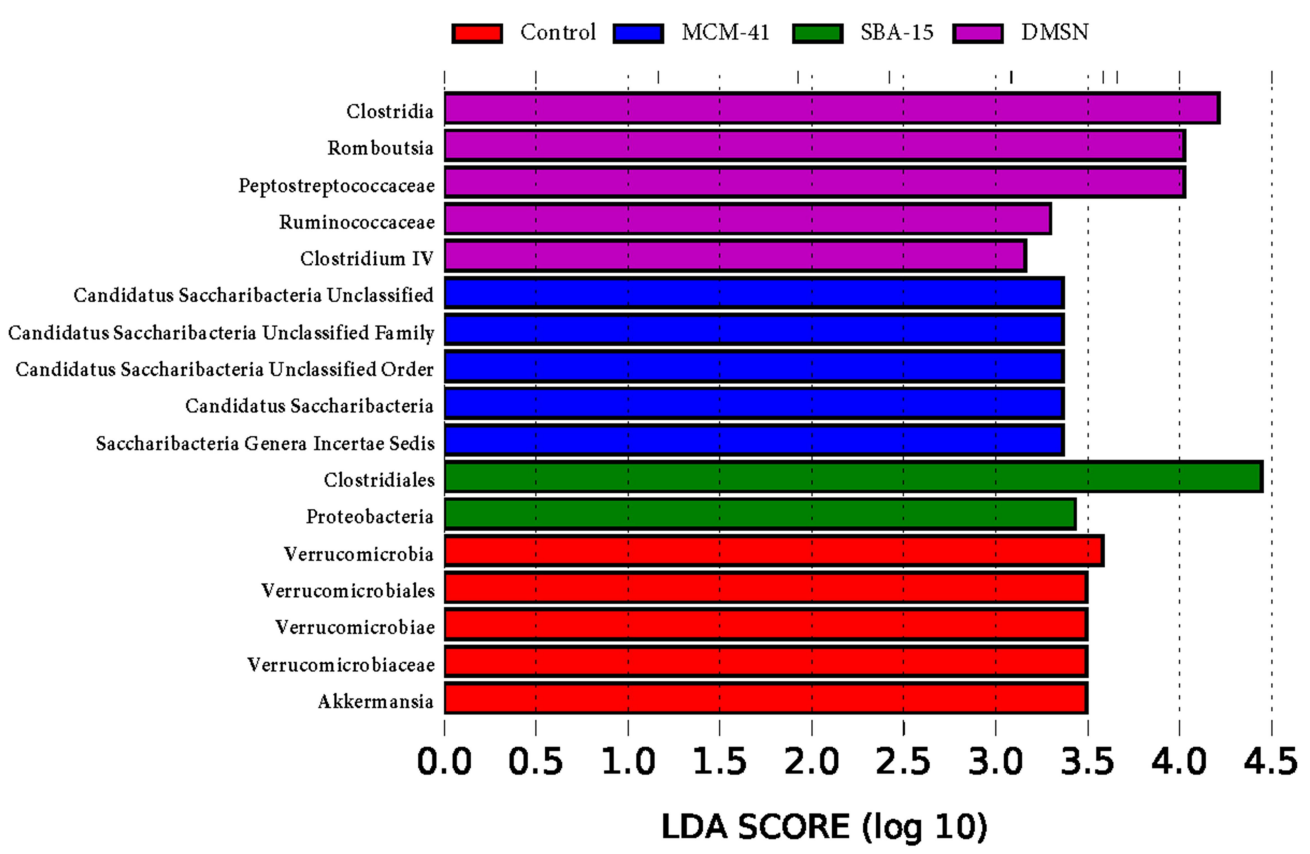

Figure 6 Specific taxa detection. Bacterial differences between (A) groups at day 7 were detected by LEfSe and (B) species with LDA score (log 10) $>3.0$ were plotted $(n=10)$. 


\section{A Verrucomicrobia}

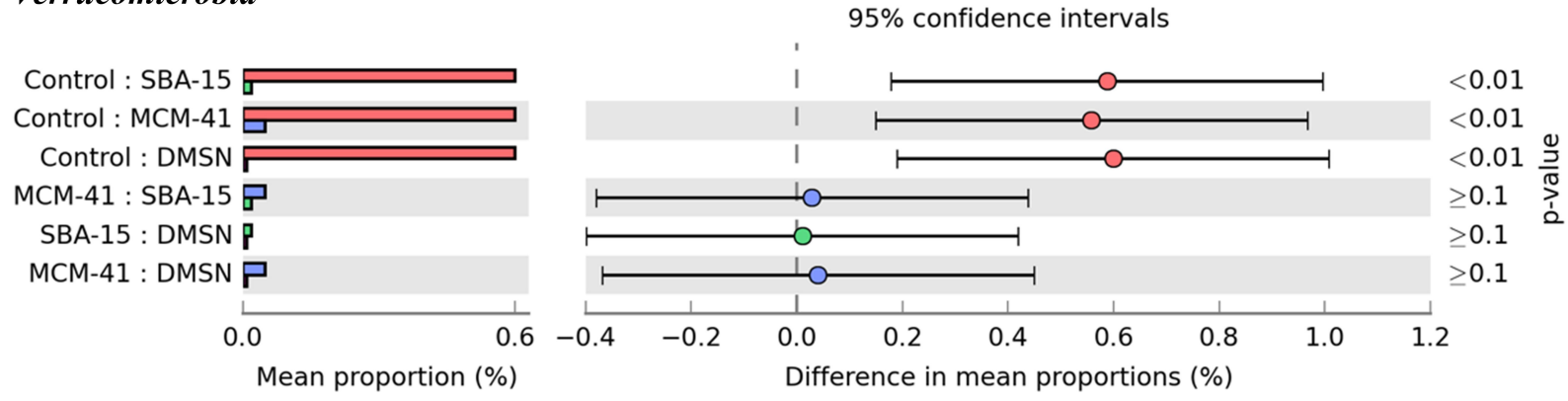

\section{B Candidatus Saccharibacteria}
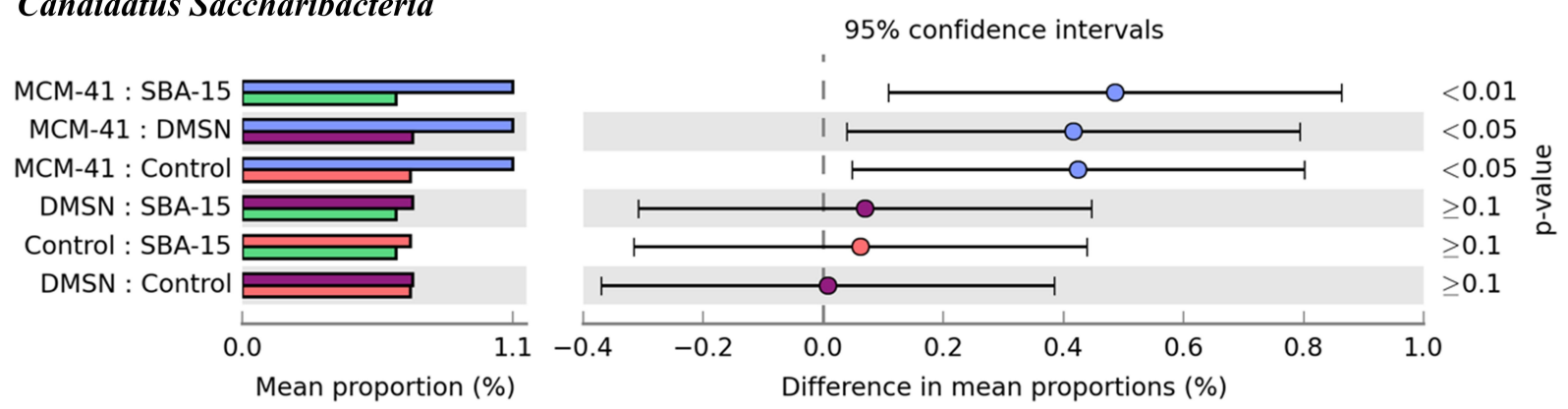

Figure 7 Multiple groups comparison at day 7. Specific phylum One-way ANOVA bar plot. (A) Verrucomicrobia: $p$ value of Control and SBA-I5<0.0I, Control and MCM$4 I<0.01$, Control and DMSN<0.0I. (B) Candidatus Saccharibacteria: $p$ value of MCM-4I and SBA-I5<0.0I, MCM-4I and DMSN $<0.05$, MCM-4I and Control<0.05.

cranberry extract, which protected mice from diet-induced obesity.

In the aspect of Candidatus Saccharibacteria, as reported, Candidatus Saccharibacteria was a phylum of gram-negative bacteria, which has been shown to be a cause of inflammatory mucosal diseases, particularly the periodontitis. Ferrari et $\mathrm{al}^{33}$ suggested that genetically determined antibiotic resistance of Candidatus Saccharibacteria contributes to early stage of inflammatory bowel disease (IBD) progression, and they also found that Candidatus Saccharibacteria could act as a promoter of inflammation by adjusting the growth condition for competing bacterial populations. Interestingly, our study showed that the alteration of gut microbiota in MCM-41 group was primarily due to the decrease of Verrucomicrobia abundance and increase of Candidatus Saccharibacteria, which further reaffirmed the potential pro-inflammation effects in MCM-41 group.

There are several potential explanations for the lack of microbial alterations reported in our study, for example, some studies reported a longer administration period, higher administration frequency, and larger administration dosage compared with our study. Additionally, Williams et $\mathrm{al}^{34}$ observed that $16 \mathrm{~S}$ rRNA gene sequence-based evaluation of microbial communities was able to detect both live and dead bacteria. Hence, although the cultivable bacterial fraction represents a portion of the total gut microbial community, the non-cultivable fraction was still as high as $60-70 \%$. In sense of this, 16S rRNA gene sequence-based evaluation might give a different result obtained from other methods like culture-based bacteria analysis.

Previous studies have attempted to elucidate the biological effects after nanoparticle exposure, but many of these efforts have simply reaffirmed how greatly nanoparticle characteristics such as surface charge, surface coating, particle shape, particle size and administration dosage might influence the biological effects. Because of these complicating variables, more work is needed to fully understand any potential adverse effects after oral nanoparticle exposure on the mammalian gastrointestinal tract. The present study complemented the toxicity and safety evaluation of MSNs from a novel and crucial perspective, the gut microbiota. However, we still face a myriad of challenges in understanding whether and how the nanoparticles cause gut microbiota turbulence. Therefore, elucidating the interactions between nanoparticles and gut microbiota remains another interesting field and undoubtedly, would be one of the most challenging difficulties in the field of nanotoxicology. This study laid a good foundation for the subsequent application of MSNs, especially for its further clinical evaluation. Besides, this study 
also opens a new avenue for the safety or toxicity evaluation of nanomaterials, which are expected of great potential in clinic.

\section{Conclusion}

Here in this passage, three different MSNs (MCM-41, SBA15 , and DMSN) were successfully prepared. The pore size of three MSNs was calculated similarly as $(3.54 \pm 0.15) \mathrm{nm}$, $(3.48 \pm 0.21) \mathrm{nm}$, and $(3.45 \pm 0.17) \mathrm{nm}$ according to the BET \& BJH model, respectively, while the particle size of MCM41, SBA-15 and DMSN was around $209.2 \mathrm{~nm}, 1349.56 \mathrm{~nm}$, and $244.4 \mathrm{~nm}$, respectively. It was firstly observed that daily orally administration with the dosage of $50 \mathrm{mg} \cdot \mathrm{kg}^{-1}$ of SBA15 and DMSN for 7 days exerted no pro-inflammatory effects on colon of rats, while MCM-41 could induce slight inflammation on colon. To clarify the reason of this proinflammatory effect, the influence of MSNs on gut microbiota was then investigated. In accordance with previous studies, ${ }^{35-37}$ Bacteroidetes and Firmicutes were shown to be the most abundant populations of the rats' gut microbiota among the 22 phyla bacterial identified in this study, and oral administration of these three MSNs showed no influence on Bacteroidetes and Firmicutes. As reported, ${ }^{38,39}$ with the decrease of Verrucomicrobia and increase of Candidatus Saccharibacteria could potentially induce inflammation. Herein, it was found that the abundance of Verrucomicrobia was found significantly decreased, and Candidatus Saccharibacteria was found significantly increased in MCM-41 treated group at day 7, which indicated the proinflammation effect of oral administration of $50 \mathrm{mg} \cdot \mathrm{kg}^{-1}$ MCM-41 was potentially through the VerrucomicrobiaCandidatus Saccharibacteria axis.

\section{Acknowledgments}

This work was supported by the Macau Science and Technology Development fund (FDCT (067/2018/A2, 033/2017/AMJ, 0007/2019/AKP, and 0052/2020/A), and National Natural Science Foundation of China (Grant No. 81973320) to Yi Zhun Zhu.

\section{Disclosure}

The authors report no conflicts of interest in this work.

\section{References}

1. Farjadian F, Ghasemi A, Gohari O, Roointan A, Karimi M, Hamblin MR. Nanopharmaceuticals and nanomedicines currently on the market: challenges and opportunities. Nanomedicine. 2019;14 (1):93-126. doi:10.2217/nnm-2018-0120
2. Zhan J, Ma Z, Wang D, et al. Magnetic and $\mathrm{pH}$ dual-responsive mesoporous silica nanocomposites for effective and low-toxic photodynamic therapy. Int J Nanomedicine. 2017; Volume 12:2733-2748. doi:10.2147/IJN.S127528

3. Taqanaki ER, Heidari R, Monfared M, Tayebi L, Azadi A, Farjadian F. EDTA-modified mesoporous silica as supra adsorbent of copper ions with novel approach as an antidote agent in copper toxicity. Int $J$ Nanomedicine. 2019;14:7781-7792. doi:10.2147/IJN. S218760

4. Mehmood Y, Khan IU, Shahzad Y, et al. In-vitro and in-vivo evaluation of velpatasvir-loaded mesoporous silica scaffolds. A prospective carrier for drug bioavailability enhancement. Pharmaceutics. 2020;12 (4):307. doi:10.3390/pharmaceutics 12040307

5. Yang G, Li Z, Wu F, et al. Improving solubility and bioavailability of breviscapine with mesoporous silica nanoparticles prepared using ultrasound-assisted solution-enhanced dispersion by supercritical fluids method. Int $J$ Nanomedicine. 2020;15:1661-1675. doi:10.2147/IJN.S238337

6. Tran VA, Vo VG, Shim K, Lee SW, An SSA. Multimodal mesoporous silica nanocarriers for dual stimuli-responsive drug release and excellent photothermal ablation of cancer cells. Int J Nanomedicine. 2020; Volume 15:7667-7685. doi:10.2147/IJN.S254344

7. Farjadian F, Roointan A, Mohammadi-Samani S, Hosseini M. Mesoporous silica nanoparticles: synthesis, pharmaceutical applications, biodistribution, and biosafety assessment. Chem Eng J. 2019.

8. Garcia-Bennett AE. Synthesis, toxicology and potential of ordered mesoporous materials in nanomedicine. Nanomedicine. 2011;6 (5):867-877. doi:10.2217/nnm.11.82

9. Fu C, Liu T, Li L, Liu H, Chen D, Tang F. The absorption, distribution, excretion and toxicity of mesoporous silica nanoparticles in mice following different exposure routes. Biomaterials. 2013;34 (10):2565-2575. doi:10.1016/j.biomaterials.2012.12.043

10. Li L, Liu T, Fu C, Tan L, Meng X, Liu H. Biodistribution, excretion, and toxicity of mesoporous silica nanoparticles after oral administration depend on their shape. Nanomedicine. 2015;11(8):1915-1924. doi:10.1016/j.nano.2015.07.004

11. Chen L, Guo Y, Hu C, Lam PKS, Lam JCW, Zhou B. Dysbiosis of gut microbiota by chronic coexposure to titanium dioxide nanoparticles and bisphenol A: implications for host health in zebrafish. Environ Pollut. 2018;234:307-317. doi:10.1016/j.envpol.2017.11.074

12. Wilding LA, Bassis CM, Walacavage $\mathrm{K}$, et al. Repeated dose (28-day) administration of silver nanoparticles of varied size and coating does not significantly alter the indigenous murine gut microbiome. Nanotoxicology. 2016;10(5):513-520. doi:10.3109/ 17435390.2015.1078854

13. Wu SH, Lin HP. Synthesis of mesoporous silica nanoparticles. Chem Soc Rev. 2013;42(9):3862-3875. doi:10.1039/c3cs35405a

14. Zhao D, Feng J, Huo Q, et al. Triblock copolymer syntheses of mesoporous silica with periodic 50 to 300 angstrom pores. Science (80-). 1998;279(5350):548-552. doi:10.1126/science.279.5350.548

15. Shen D, Yang J, Li X, et al. Biphase stratification approach to three-dimensional dendritic biodegradable mesoporous silica nanospheres. Nano Lett. 2014;14(2):923-932. doi:10.1021/n1404316v

16. Köchling T, Sanz JL, Gavazza S, Florencio L. Analysis of microbial community structure and composition in leachates from a young landfill by 454 pyrosequencing. Appl Microbiol Biotechnol. 2015;99 (13):5657-5668. doi:10.1007/s00253-015-6409-4

17. Kozich JJ, Westcott SL, Baxter NT, Highlander SK, Schloss PD. Development of a dual-index sequencing strategy and curation pipeline for analyzing amplicon sequence data on the miseq illumina sequencing platform. Appl Environ Microbiol. 2013;79 (17):5112-5120. doi:10.1128/AEM.01043-13

18. Martínez-Carmona M, Gun'ko YK, Vallet-Regí M. Mesoporous silica materials as drug delivery: "the nightmare" of bacterial infection. Pharmaceutics. 2018;10(4):279. doi:10.3390/pharmaceutics10 040279 
19. González B, Colilla M, Díez J, et al. Mesoporous silica nanoparticles decorated with polycationic dendrimers for infection treatment. Acta Biomater. 2018;68:261-271. doi:10.1016/j.actbio.2017.12.041

20. Mohammadi H, Seyyed RH, Niknezhad V, Jamshidzadeh A, Farjadian F. In vitro and in vivo evaluation of succinic acid-substituted mesoporous silica for ammonia adsorption: potential application in the management of hepatic encephalopathy. Int $J$ Nanomedicine. 2020;15:10085-10098. doi:10.2147/IJN.S271883

21. Dogra P, Adolphi NL, Wang Z, et al. Establishing the effects of mesoporous silica nanoparticle properties on in vivo disposition using imaging-based pharmacokinetics. Nat Commun. 2018;9(1). doi:10.1038/s41467-018-06730-z.

22. Yu T, Hubbard D, Ray A, Ghandehari H. In vivo biodistribution and pharmacokinetics of silica nanoparticles as a function of geometry, porosity and surface characteristics. J Control Release. 2012;163 (1):46-54. doi:10.1016/j.jconrel.2012.05.046

23. Yu T, Malugin A, Ghandehari H. Impact of silica nanoparticle design on cellular toxicity and hemolytic activity. ACS Nano. 2011;5 (7):5717-5728. doi:10.1021/nn2013904

24. Hong X, Zhong X, Du G, et al. The pore size of mesoporous silica nanoparticles regulates their antigen delivery efficiency. Sci Adv. 2020;6(25):eaaz4462. doi:10.1126/sciadv.aaz4462

25. Huang X, Li L, Liu T, et al. The shape effect of mesoporous silica nanoparticles on biodistribution, clearance, and biocompatibility in vivo. ACS Nano. 2011;5(7):5390-5399. doi:10.1021/nn200365a

26. He Q, Zhang Z, Gao F, Li Y, Shi J. In vivo biodistribution and urinary excretion of mesoporous silica nanoparticles: effects of particle size and PEGylation. Small. 2011;7(2):271-280. doi:10.1002/ smll.201001459

27. Clarridge JE. Impact of $16 \mathrm{~S}$ rRNA gene sequence analysis for identification of bacteria on clinical microbiology and infectious diseases. Clin Microbiol Rev. 2004;17(4):840-862. doi:10.1128/ CMR.17.4.840-862.2004

28. Caporaso JG, Lauber CL, Walters WA, et al. Global patterns of $16 \mathrm{~S}$ rRNA diversity at a depth of millions of sequences per sample. Proc Natl Acad Sci U S A. 2011;108(Supplement_1):4516-4522. doi:10.1073/pnas.1000080107

29. Langille MGI, Zaneveld J, Caporaso JG, et al. Predictive functional profiling of microbial communities using 16S rRNA marker gene sequences. Nat Biotechnol. 2013;31(9):814-821. doi:10.1038/ nbt. 2676
30. Chauhan S, Manivasagam G, Kumar P, Ambasta RK. Cellular toxicity of mesoporous silica nanoparticle in SHSY5Y and BMMNCs cell. Pharm Nanotechnol. 2018;6(4):245-252. doi:10.2174/ 2211738506666181031160108

31. Tarn D, Ashley CE, Xue M, Carnes EC, Zink JI, Brinker CJ. Mesoporous silica nanoparticle nanocarriers: biofunctionality and biocompatibility. Acc Chem Res. 2013;46(3):792-801. doi:10.1021/ ar3000986

32. Lehman SE, Morris AS, Mueller PS, Salem AK, Grassian VH, Larsen SC. Silica nanoparticle-generated ROS as a predictor of cellular toxicity: mechanistic insights and safety by design. Environ Sci Nano. 2016;3(1):56-66. doi:10.1039/C5EN00179J

33. Ferrari B, Winsley T, Ji M, Neilan B. Insights into the distribution and abundance of the ubiquitous candidatus Saccharibacteria phylum following tag pyrosequencing. Sci Rep. 2014;4. doi:10.1038/ srep03957

34. Williams K, Milner J, Boudreau MD, Gokulan K, Cerniglia CE, Khare S. Effects of subchronic exposure of silver nanoparticles on intestinal microbiota and gut-associated immune responses in the ileum of Sprague-Dawley rats. Nanotoxicology. 2015;9(3):279-289. doi: $10.3109 / 17435390.2014 .921346$

35. Pan H, Guo R, Zhu J, et al. A gene catalogue of the Sprague-Dawley rat gut metagenome. Gigascience. 2018;7(5). doi:10.1093/gigascience/giy055.

36. Paranjpe M, Müller-Goymann CC. Nanoparticle-mediated pulmonary drug delivery: a review. Int J Mol Sci. 2014;15(4):5852-5873. doi:10.3390/ijms15045852

37. Li XX, Shi S, Rong L, Feng MQ, Zhong L. The impact of liposomal linolenic acid on gastrointestinal microbiota in mice. Int J Nanomedicine. 2018;13:1399-1409. doi:10.2147/IJN.S151825

38. Kuehbacher T, Rehman A, Lepage P, et al. Intestinal TM7 bacterial phylogenies in active inflammatory bowel disease. J Med Microbiol. 2008;57(12):1569-1576. doi:10.1099/jmm.0.47719-0

39. Fujio-Vejar S, Vasquez Y, Morales P, et al. The gut microbiota of healthy chilean subjects reveals a high abundance of the phylum verrucomicrobia. Front Microbiol. 2017;8. doi:10.3389/ fmicb.2017.01221
International Journal of Nanomedicine

\section{Publish your work in this journal}

The International Journal of Nanomedicine is an international, peerreviewed journal focusing on the application of nanotechnology in diagnostics, therapeutics, and drug delivery systems throughout the biomedical field. This journal is indexed on PubMed Central, MedLine, CAS, SciSearch ${ }^{\circledR}$, Current Contents ${ }^{\circledR} /$ Clinical Medicine,
Journal Citation Reports/Science Edition, EMBase, Scopus and the Elsevier Bibliographic databases. The manuscript management system is completely online and includes a very quick and fair peer-review system, which is all easy to use. Visit http://www.dovepress.com/ testimonials.php to read real quotes from published authors. 